\title{
EFEITOS DE SURFATANTES SOBRE A TENSÃO SUPERFICIAL E A ÁREA DE MOLHAMENTO DE SOLUÇÕES DE GLYPHOSATE SOBRE FOLHAS DE TIRIRICA ${ }^{1}$
}

\author{
CRISTIANE G. DE MENDONÇA ${ }^{2}$, EDIVALDO D. VELINI ${ }^{3}$, DAGOBERTO MARTINS ${ }^{3}$ e CRISTINA G. DE MENDONÇA ${ }^{2}$
}

\section{RESUMO}

Com o objetivo de avaliar a eficiência da agregação de surfatantes ao herbicida glyphosate analisou-se a tensão superficial de diferentes soluções de pulverização contendo o hebicida e o surfatante, e a área de molhamento destas soluções nas folhas de Cyperus rotundus L.. Foram desenvolvidos métodos para avaliação da tensão superficial e da área de molhamento. Para analisar a tensão fez-se pesagens das gotas formadas na extremidade de uma bureta, com os seguintes tratamentos combinados de forma fatorial $(3 \times 5 \times$ 11): 3 surfatantes (Extravon, Aterbane e Silwet L77), 5 concentrações do herbicida, produto comercial Roundup $(0 ; 1 ; 2 ; 3,5$ e $5 \%)$ e 11 concentrações de cada surfatantes $(0 ; 0,005 ; 0,01$; 0,$02 ; 0,05 ; 0,1 ; 0,2 ; 0,5 ; 1 ; 2$ e $3,5 \%$ ), num total de 165 tratamentos. Para avaliar a área de molhamento nas folhas de tiririca aplicou-se gotas de $0,48 \mu \mathrm{l}$. Os dados foram ajustados pelo modelo de Mitscherlich e, observou-se que para o surfatante Extravon que a eficiência decrescia gradativamente a medida em que aumentava a concentração do herbicida; para o Aterbane a eficiência foi reduzida apenas em baixas concentrações; já o surfatante Silwet L-77 apresentou eficiência bem superior aos demais e sua eficiência foi pouco alterada com a adição herbicida. Houve uma correlação positiva entre área de molhamento e tensão superficial. Concluiu-se, ainda, que não basta um surfatante reduzir a tensão superficial da água destilada, para que possa ser recomendado seu uso agrícola, assim, o surfatante deve ser submetido a testes preliminares com os defensivos em que serão conjugados para posterior recomendação.

Palavras chave: Surfatantes, herbicida, tecnologia de aplicação, método experimental, Cyperus rotundus.

\section{ABSTRACT \\ Effects of surfactants on surface tension and foliar wetting solutions of glyphosate over purple nutsedge leaves}

This research was undertaken to develop methods to evaluate the surface tension and foliar wetting of surfactant added in herbicide. It used three surfactants (Extravon, Aterbane and Silwet), five concentration of herbicide and eleven surfactant concentrations each. Surface tension and foliar wetting were evaluated by drops weight and sprayed drops with $0,48 \mu$ l of solution over
Cyperus rotundus leaves. The surface tension was decreased glyphosate added. Silwet was the best of surfactants to reduction on surface tension and increased on foliar wetting.

Key words: Surfactant, Cyperus rotundus, herbicide, experimental method, technology applyed.

\footnotetext{
${ }^{1}$ Recebido para publicação em 23/07/98 e na forma revisada em 05/01/2000.

${ }^{2} \mathrm{Eng}^{\mathrm{o}} \mathrm{Agr}^{\mathrm{\rho}}$, Dept ${ }^{\mathrm{o}}$ de Produção Vegetal da FCA/UNESP, C.P. 237, CEP: 18603-970, Botucatu/SP, Brasil.

${ }^{3}$ Prof $^{\circ}$ Assistente Doutor, Dept ${ }^{\circ}$ de Produção Vegetal, FCA/UNESP.
} 


\section{INTRODUÇÃO}

O glyphosate consiste-se no herbicida de mais ampla utilização em todo mundo. Contribuem para esta posição de destaque, sua elevada eficiência, amplo espectro de controle, grande capacidade de translocação, baixo custo, rápida dissipação no meio ambiente, elevada eficiência e baixa toxidez a humanos, animais domésticos e silvestres. Algumas estimativas laboratoriais da tensão superficial e da adesão da calda do herbicida às folhas das plantas daninhas podem substituir um grande número de parcelas experimentais e complexos procedimentos de lavagem das folhas, com o intuito de selecionar inicialmente surfatantes e as doses que podem aumentar a eficiência de herbicidas em geral, destacando-se o glyphosate.

A agregação de surfatantes à calda de aplicação do glyphosate tem-se tornado comum, com o intuito de aumentar a eficiência, reduzir o período livre de chuva e a dose do glyphosate (Reddy \& Singh, 1992; Baylis \& Hart, 1993; Sun et al., 1996). O aumento da ação dos herbicidas pela adição dos surfatantes pode permitir uma redução de dose ou promover eficácia contra espécies de difícil controle e este aumento de eficiência está relacionado com o aumento da molhabilidade da superfície, redução de tensão superficial e ângulo de contato da gota, além de aumentar a penetração cuticular Singh (1993).

A tensão superficial é um dos fatores chaves na performance dos surfatantes e sua redução é mais importante do que o tamanho da gota, velocidade da gota e ângulo de incidência na folha para a determinação da adesão nas folha (Stevens et al., 1993).

O mecanismo de ação dos surfatantes na superfície das folhas está correlacionado com o aumento da área de molhamento, dissolvemento ou na desruptura das ceras epicuticulares, solubilidade dos agroquímicos, prevenção ou atraso na formação de cristais do depósito, retenção de umidade pela sua ação umectante e aumento da absorção das soluções via infiltração estomatal (Stock \& Holloway, 1993). A redução da tensão superficial a 28-32 dina/cm induzida por surfatantes leva a uma penetração estomatal em folhas de Pyrus communis L. (Greene \& Bucovac, 1974).

A capacidade de molhamento dos surfatantes são atribuídas a sua estrutura molecular, a sua afinidade à substâncias hidrofóbicas e sua habilidade em abaixar a tensão superficial da solução (Goddard \& Padmanabhan, 1992). E sua eficiência é dependente da natureza do herbicida, da espécie de planta daninha e das condições ambientais (Roeggenbuck et al., 1993). Chung \& Kwon (1992) sugere que o molhamento da superfície foliar varia com o grau de cobertura da cera de variedades de arroz do que das diferenças morfológicas sutis da superfície foliar.

O objetivo do presente trabalho foi avaliar a eficiência da mistura de surfatantes ao herbicida glyphosate sobre a tensão supreficial de soluções e área de molhamento de folhas de Cyperus rotundus $\mathrm{L}$.

\section{MATERIAIS E MÉTODOS}

Para o preparo das soluções foram utilizados o herbicida glyphosate (produto comercial Roundup) e os surfatantes: Extravon, Aterbane e Silwet L-77, cujo ingredientes ativos são aquil-fenol-poliglicóleter, condensado de alcoolfenóis com óxido de eteno e sulfonados orgânicos e copolímero silicone-poliether, respetivamente.

Para determinar da tensão superficial foram preparadas soluções de glyphosate (produto comercial Roundup) agregando-se os surfatantes combinados de forma fatorial: 5 concentrações do herbicida $(0 ; 1.0 ; 2.0 ; 3.5$ e $5.0 \%(\mathrm{v} / \mathrm{v})$ do produto comercial Roundup), os 3 tipos de surfatantes em 11 concentrações de cada surfatante, num total de 165 tratamentos (Tabela 1).

Estimou-se a tensão superficial dos tratamentos medindo-se o peso das gotas formadas na extremidade da bureta, em balança de precisão com quatro casas decimais em gramas, 
num tempo aproximado de 30 segundos. Cada gota pesada correspondeu a uma repetição, num total de 15 repetições. Para evitar perdas por evaporação usou-se uma camada de óleo no becker de $25 \mathrm{ml}$ colocado sobre a balança. As buretas utilizadas foram calibradas através do tratamento com água destilada.

TABELA 1. Tratamentos utilizados na determinação da tensão superficial.

\begin{tabular}{|c|c|c|c|c|}
\hline \multicolumn{3}{|c|}{ Surfatantes } & \multirow{2}{*}{$\begin{array}{c}\text { Concentrações } \\
\text { dos Surfatantes } \\
(\mathrm{v} / \mathrm{v})\end{array}$} & \multirow{2}{*}{$\begin{array}{c}\text { Concentrações } \\
\text { de Roundup } \\
\text { (Glyphosate, v/v) }\end{array}$} \\
\hline $\begin{array}{c}\text { Produto } \\
\text { Comercial } \\
\end{array}$ & $\begin{array}{c}\text { Ingrediente } \\
\text { ativo (i.a.) }\end{array}$ & $\begin{array}{c}\text { Conc. do i.a. } \\
(\mathrm{g} / \mathrm{l})\end{array}$ & & \\
\hline Extravon & $\begin{array}{l}\text { alquil-fenol- } \\
\text { poliglicóleter }\end{array}$ & 250 & $\begin{array}{c}0 ; 0,005 ; 0,01 ; \\
0,02 ; 0,05 ; 0,10 ; \\
0,20 ; 0,50 ; 1,0 ; 2,0 \\
\text { e } 3,0 \% \\
\end{array}$ & $\begin{array}{l}0 ; 1.0 ; 2.0 \\
3.5 \text { e } 5.0 \%\end{array}$ \\
\hline Aterbane & $\begin{array}{c}\text { condensado de } \\
\text { alcoofenóis com óxido de } \\
\text { eteno e sulfonados } \\
\text { orgânicos } \\
\end{array}$ & 450 & $\begin{array}{c}0 ; 0,005 ; 0,01 ; \\
0,02 ; 0,05 ; 0,10 ; \\
0,20 ; 0,50 ; 1,0 ; 2,0 \\
\text { e } 3,0 \% \\
\end{array}$ & $\begin{array}{l}0 ; 1.0 ; 2.0 \\
3.5 \text { e } 5.0 \%\end{array}$ \\
\hline Silwet L-77 & $\begin{array}{l}\text { copolímero silicone- } \\
\text { polieter }\end{array}$ & 77 & $\begin{array}{c}0 ; 0,005 ; 0,01 ; \\
0,02 ; 0,05 ; 0,10 ; \\
0,20 ; 0,50 ; 1,0 ; 2,0 \\
\text { e } 3,0 \%\end{array}$ & $\begin{array}{l}0 ; 1.0 ; 2.0 \\
3.5 \text { e } 5.0 \%\end{array}$ \\
\hline
\end{tabular}

O método utilizado para determinação da área de molhamento foi uma adaptação do procedimento utilizado por Kadota \& Matsunaka (1986) o que permitiu determinar a área de molhamento quando gotas da calda herbicida são depositadas sobre as folhas das plantas daninhas.

Para avaliação da área de molhamento foram selecionadas através de análises da tensão superficial as seguintes concentrações dos surfatantes em soluções de $2 \%$ v/v de Roundup: para Extravon: 0,05\%; 0,2\%; 1,0\%; 3,0\% v/v; para Aterbane: $0,2 \% ; 0,1 \% ; 1,0 \% ; 3,0 \% \mathrm{v} / \mathrm{v}$; para Silwet L-77: $0,005 \% ; 0,02 \% ; 0,1 \% ; 1,0 \% ; 3,0 \%$ v/v e os tratamentos com Roundup sozinho.

O método utilizado foi a deposição de gotas de $0,48 \mu \mathrm{l}$ sobre a face adaxial de folhas maduras de $C$. rotundus (tiririca), utilizando 6 repetições. As folhas de tiririca foram fixadas de forma firme em pedaços de madeira $(9,0 \times 1,5 \times$ $2,0 \mathrm{~cm}) \mathrm{com}$ fita dupla adesiva no sentido do comprimento e com o auxílio de uma microseringa colocou-se a gota sobre a folha.

Para melhorar a visualização da área de molhamento através da lupa, foi necessário borrifar amido (penetrose) de forma delicada, apenas para fixar a gota na folha.

As áreas de molhamento foram obtidas através de desenhos, em folhas de transparências fixadas na lupa. Pesou-se um pedaço de transparência com dimensões conhecidas e pesouse os desenhos formados pelas áreas de molhamento e por relação obteve-se a área de molhamento em $\mathrm{cm}^{2}$.

Os dados obtidos foram submetidos a análise de variância pelo Teste $\mathrm{F}$ e efetuou-se regressões com o auxílio do programa SAS. 


\section{RESULTADOS E DISCUSSÃO}

Na Figura 1 está representado o modelo de MITSCHERLICH utilizado nas análises de regressão. Para que o modelo ajustasse aos dados, houve a necessidade de modificá-lo. Os modelos original e simplificado são descritos a seguir e representados na Figura 1.

Modelo Original: $\mathrm{Y}=\mathrm{A} \times\left(1-10^{-\mathrm{C} \times(\mathrm{X}+\mathrm{B})}\right)$

Modelo Utilizado: $\mathrm{Y}=\mathrm{T}_{\text {água }}-\mathrm{A} \times\left(1-10^{-\mathrm{C} \times(\mathrm{X}+\mathrm{B})}\right)$ onde:

$$
\begin{aligned}
\mathrm{Y}= & \text { Tensão superficial, em } \mathrm{N} / \mathrm{m} ; \\
\mathrm{A}= & \text { Assíntota horizontal máxima no modelo } \\
& \text { original; } \\
\mathrm{C}= & \text { Concavidade da curva; } \\
\mathrm{B}= & \text { Ponto de interceptação do eixo abcissas; } \\
\mathrm{T}_{\text {água }}= & 0,0726 \mathrm{~N} / \mathrm{m} ; \\
\mathrm{X}= & \text { Concentração do sufartante, em } \\
& \text { porcentagem; } \\
\mathrm{T}_{\text {água }}-\mathrm{A}= & \text { Assíntota horizontal mínima no } \\
& \text { modelo utilizado. }
\end{aligned}
$$

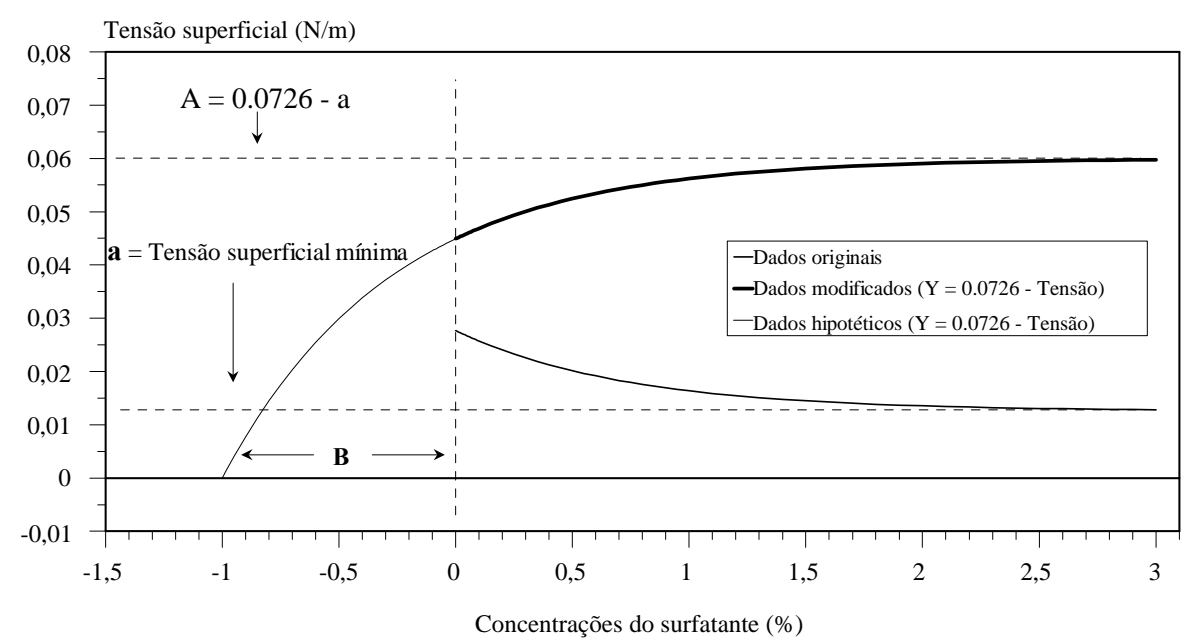

FIGURA 1. Caracterização do modelo utilizado nas análises de regressão. Os dados de tensão foram modificados para que fosse possível o uso do modelo de Mitscherlich, que tem A e B como parâmetros e $\mathrm{C}$ a concavidade da curva.

Todos os elementos da equação tem seu significado prático; a expressão "Tágua - A" corresponde à mínima tensão superficial que pode ser alcançada com o uso do surfatante em questão. O parâmetro "C" representa a eficiência do surfatante, e o parâmetro " $\mathrm{B}$ " indica quanto do surfatante tem que colocar para que se obtenha a mesma redução de tensão condicionada pela adição do herbicida na concentração considerada.

Na Tabela 2 estão descritos os parâmetros obtidos na regressão pelo modelo de MITSCHERLICH, nas diferentes concentrações de glyphosate (produto comercial Roundup) para os surfatantes Extravon, Aterbane e Silwet L-77. Pela concavidade da curva (parâmetro C) da Tabela 2 concluiu-se que o Silwet L-77 tem um coeficiente de eficácia melhor que o Aterbane e este melhor que o Extravon, quando comparados em cada concentração do herbicidas e esta eficácia decresce com o aumento da concentração do herbicida para todos surfatantes. Este valor reflete a capacidade da solução em atingir a tensão superficial mínima numa menor concentração de surfatante.

A eficiência do surfatante Extravon é reduzida quando ocorre interação com o herbicida Roundup, já que sua tensão superficial mínima é maior conforme o aumento da concentração do herbicida.

O comportamento sinérgico do surfatante Aterbane em adição com glyphosate a altas concentrações mostra uma redução e estabilização 
da tensão superficial mínima na presença do herbicida. O surfatante Aterbane perde parte de sua eficiência quando em baixas concentrações e associados ao glyphosate, mas em altas concentrações do surfatante e associado ao glyphosate é mais eficiente quando comparado com surfatante Aterbane em água. Assim, o surfatante Aterbane sofre menor influência com relação a redução da eficiência quando colocado com herbicida glyphosate (produto comercial Roundup).

TABELA 2. Resultados das análises de variância e regressão. Parâmetros das equações obtidas.

\begin{tabular}{|c|c|c|c|c|}
\hline \multirow{2}{*}{$\begin{array}{c}\text { Concentrações } \\
\text { de Roundup }\end{array}$} & \multirow{2}{*}{$\begin{array}{c}\text { Parâmetros do modelo } \\
\text { de Mitscherlich }\end{array}$} & \multicolumn{3}{|c|}{ Surfatantes } \\
\hline & & Extravon & Aterbane & Silwet L-77 \\
\hline \multirow{6}{*}{0} & A & 0,04119 & 0,04015 & 0,05372 \\
\hline & B & 0 & 0 & 0 \\
\hline & $\mathrm{C}$ & 39,62 & 44,46 & 86,26 \\
\hline & Tensão mínima & 0,03140 & 0,03244 & 0,01887 \\
\hline & $\mathrm{F}$ & $2863855,05^{* *}$ & $1412393,93 * *$ & $4057355,76^{* *}$ \\
\hline & $r^{2}$ & 0,9974 & 0,9816 & 0,9974 \\
\hline \multirow{6}{*}{1} & $\mathrm{~A}$ & 0,04015 & 0,04312 & 0,05481 \\
\hline & B & 0,31774 & 0,07590 & 0,02990 \\
\hline & $\mathrm{C}$ & 1,476 & 5,381 & 11,723 \\
\hline & Tensão mínima & 0,03244 & 0,02947 & 0,01778 \\
\hline & $\mathrm{F}$ & $255118,37 * *$ & $191162,9 * *$ & $636018,66^{* *}$ \\
\hline & $r^{2}$ & 0,9927 & 0,9970 & 0,9315 \\
\hline \multirow{6}{*}{2} & $\mathrm{~A}$ & 0,03943 & 0,04318 & 0,05267 \\
\hline & B & 0,56839 & 0,12569 & 0,02340 \\
\hline & $\mathrm{C}$ & 0,857 & 3,202 & 14,134 \\
\hline & Tensão mínima & 0,03316 & 0,02941 & 0,01992 \\
\hline & $\mathrm{F}$ & $208628,1^{* *}$ & $203476,88 * *$ & $702173,01 * *$ \\
\hline & $r^{2}$ & 0,9925 & 0,9960 & 0,9703 \\
\hline \multirow{6}{*}{3.5} & $\mathrm{~A}$ & 0,03934 & 0,04316 & 0,05314 \\
\hline & B & 0,92303 & 0,21330 & 0,05490 \\
\hline & $\mathrm{C}$ & 0,533 & 1,978 & 6,812 \\
\hline & Tensão mínima & 0,03325 & 0,02943 & 0,01945 \\
\hline & $\mathrm{F}$ & $148661,29 * *$ & $181081,29 * *$ & $655994,71 * *$ \\
\hline & $r^{2}$ & 0,9937 & 0,9923 & 0,9610 \\
\hline \multirow{6}{*}{5} & A & 0,03882 & 0,04305 & 0,05217 \\
\hline & B & 1,07086 & 0,28885 & 0,06356 \\
\hline & $\mathrm{C}$ & 0,476 & 1,463 & 5,863 \\
\hline & Tensão mínima & 0,03377 & 0,02954 & 0,02042 \\
\hline & $\mathrm{F}$ & $171783,98 * *$ & $186772,88 * *$ & $639625,28 * *$ \\
\hline & $r^{2}$ & 0,9974 & 0,9950 & 0,9580 \\
\hline \multicolumn{5}{|l|}{ Valores de F } \\
\hline $\mathrm{G}$ & & 16441,67 & 20596,31 & 21413,09 \\
\hline $\mathrm{E}$ & & 284921,00 & 233878,50 & 831973,00 \\
\hline $\mathrm{G}^{*} \mathrm{E}$ & & 34019,42 & 19338,17 & 24943,79 \\
\hline $\mathrm{E} \mathrm{d} \mathrm{G}{ }_{0}$ & & 287146,00 & 143888,50 & 406820,00 \\
\hline $\mathrm{Ed} \mathrm{G}{ }_{1}$ & & 43705,33 & 46899,44 & 136556,00 \\
\hline $\mathrm{Ed} \mathrm{G}$ & & 35748,72 & 49968,74 & 144727,00 \\
\hline $\mathrm{E} \mathrm{d} \mathrm{G}_{3.5}$ & & 25441,62 & 44631,50 & 136527,00 \\
\hline $\mathrm{Ed} \mathrm{G} \mathrm{d}_{5}$ & & 29290,09 & 45912,79 & 133530,00 \\
\hline C.V. \% & & 0,264763 & 0,335135 & 0,408959 \\
\hline
\end{tabular}

**Significativo a nível de $1 \%$ de probalidade. 
O surfatante organosilicone Silwet L-77 tem a tensão superficial mínima atingida a $1 \%$ de Roundup, seguida do surfatante isolado. Nas concentrações $2 \%, 3,5 \%$ e $5 \%$ de Roundup, há um aumento da tensão superficial mínima, mostrando um efeito antagônico. As tensões superficiais dos tratamentos de glyphosate com Silwet L-77 comprovam que a eficiência do surfatantes não é reduzida substancialmente quando junto com o herbicida glyphosate.

A tensão superficial mínima do Silwet L77 em água destilada foi $18,87 \mathrm{mN} / \mathrm{m}$, um pouco abaixo da tensão conseguida por Buick et al. (1993) que foi 22,8 mN/m. O Silwet L-77 tem um bom coeficiente de eficácia, reduzindo rápido a tensão superficial, corroborando com os resultados obtidos por Stevens et al. (1993).

O comportamento dos três surfatantes, utilizados no trabalho, sem a associação do glyphosate pode ser observados na Figura 2. O surfatante Silwet L-77 tem uma maior eficiência seguido do surfatante Extravon e Aterbane colocados em ordem de eficiência.

O surfatante Aterbane tem maior eficiência quando associado ao glyphosate, nas concentrações $1 \%, 2 \%, 3,5 \%$ e $5 \%$ do produto comercial Roundup, que o surfatante Extravon. O surfatante Extravon tem a tensão superficial influenciada negativamente quando associado ao glyphosate. Mas está bem claro nas Figuras 2, 3, 4, e 5, que o surfatante Silwet tem ótima eficiência na diminuição da tensão superficial.

Os valores de "F" das análises de variância são apresentados na Tabela 2 , os efeitos de concentrações de glyphosate, dos surfatantes e a interação entre ambos foram sempre significativos ao nível de $1 \%$ de probabilidade.

A análise da área de molhamento e tensão superficial está representada na Figura 7, esta curva acompanha o modelo de MITSCHERLICH. Observa-se que, embora a relação não seja linear, é possível prever com grande precisão a área de molhamento em função da tensão superficial da solução em estudo utilizando este modelo; o coeficiente de determinação $\left(\mathrm{r}^{2}\right)$ foi de 0,94 .
A redução da tensão superficial leva a um aumento da área de molhamento em folhas de $C$. rotundus (Tabela 3). Chung \& Kwon (1992), também concluíram que a redução da tensão superficial promove aumento do molhamento de soluções de pulverização.

Os níveis mínimos de tensão superficiais alcançados pelos surfatantes Extravon, Aterbane, Silwet e glyphosate isoladamente em função da área de molhamento podem ser observados na Figura 8. Em termos de tensão superficial e, consequentemente, área de molhamento, os produtos testados podem ser dispostos na seguinte ordem crescente em termos de eficiência: glyphosate isoladamente $<$ glyphosate + Extravon $<$ glyphosate + Aterbane $<$ glyphosate + Silwet.

A eficiência dos surfatantes Extravon, Aterbane e Silwet foi reduzida pela adição de glyphosate. A redução de eficiência foi proporcional à concentração do herbicida.

A avaliação da tensão superficial de soluções confeccionadas exclusivamente com os surfatantes apresenta pouca utilidade para definir o potencial de uso dos mesmos em condições práticas em que devem ser adicionados a soluções de herbicidas.

O surfatantes Silwet L-77 mostrou-se o mais eficiente, dentre os testados, tanto em termos de redução da tensão superficial quanto em termos de aumento da área de molhamento de folhas de C. rotundus, Singh (1993) também observou que os adjuvantes organosiliconados foram mais eficientes na redução de tensão superficial. O espalhante menos eficiente foi o Extravon e, o Aterbane apresentou comportamento intermediário.

O trabalho apresentou resultados significativos e de elevada qualidade (os valores de $r^{2}$ foram sempre superiores a 0,99), comprovando a adequação das metodologias empregadas aos propósitos do trabalho. Todos os "F" foram significativos à nível de $1 \%$ de probabilidade. Os valores de $\mathrm{r}^{2}$ são altos, indicando que os dados originais ajustam-se bem ao modelo de MITSCHERLICH. 


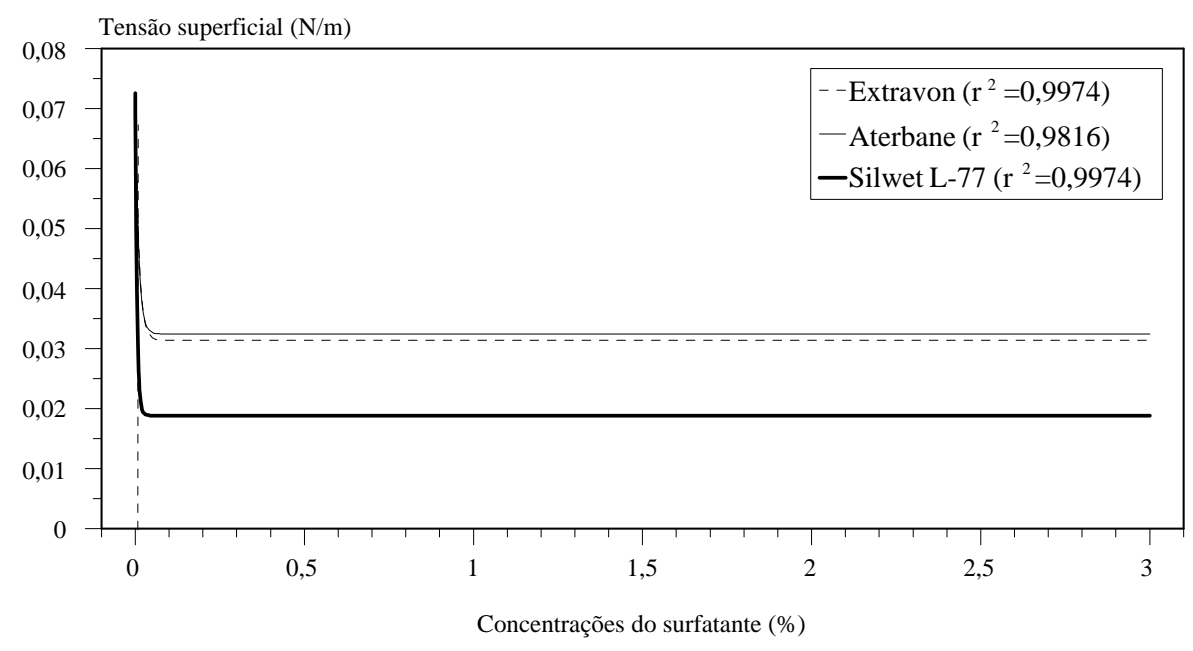

FIGURA 2. Tensões superficiais em função da concentração dos surfatantes Extravon, Aterbane e Silwet L-77. Concentração de glyphosate: $0 \%$ do produto comercial Roundup. Estimativas segundo o modelo de Mitscherlich.

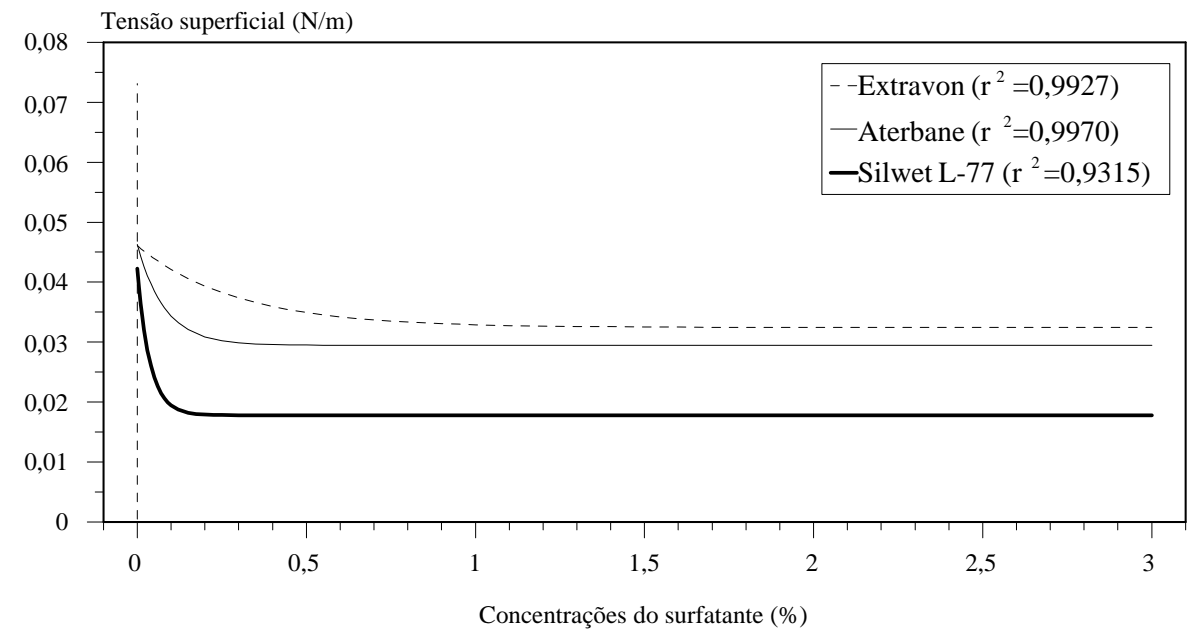

FIGURA 3. Tensões superficiais em função da concentração dos surfatantes Extravon, Aterbane e Silwet L-77. Concentração de glyphosate: $1 \%$ do produto comercial Roundup. Estimativas segundo o modelo de Mitscherlich. 


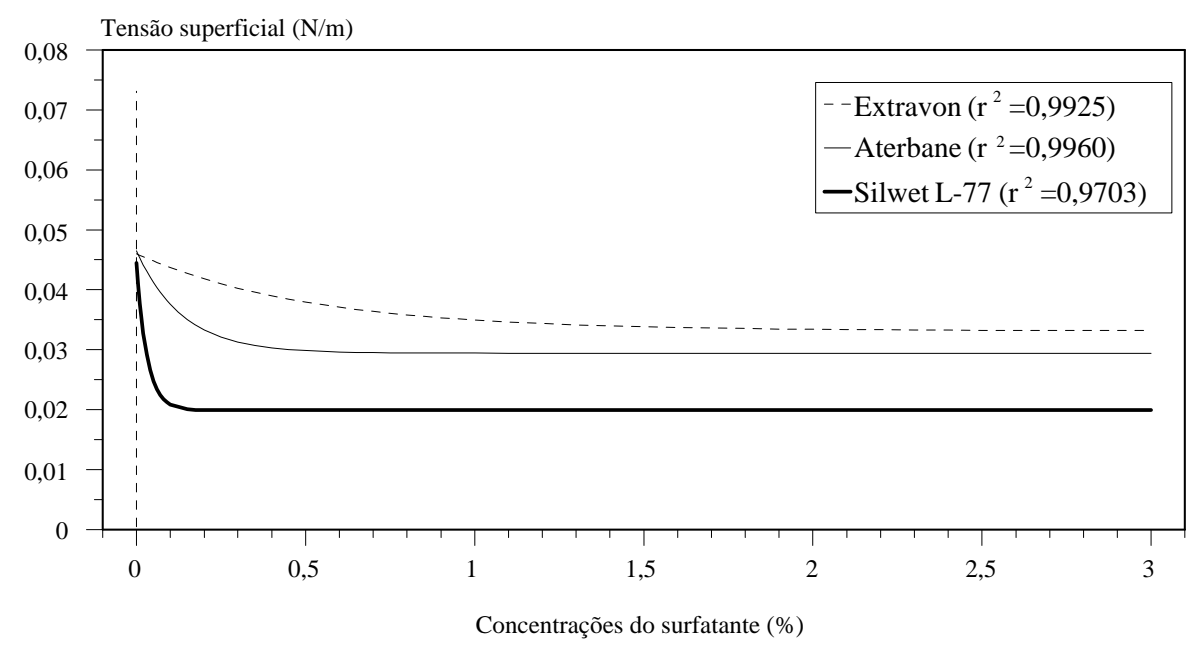

FIGURA 4. Tensões superficiais em função da concentração dos surfatantes Extravon, Aterbane e Silwet L-77. Concentração de glyphosate: $2 \%$ do produto comercial Roundup. Estimativas segundo o modelo de Mitscherlich.

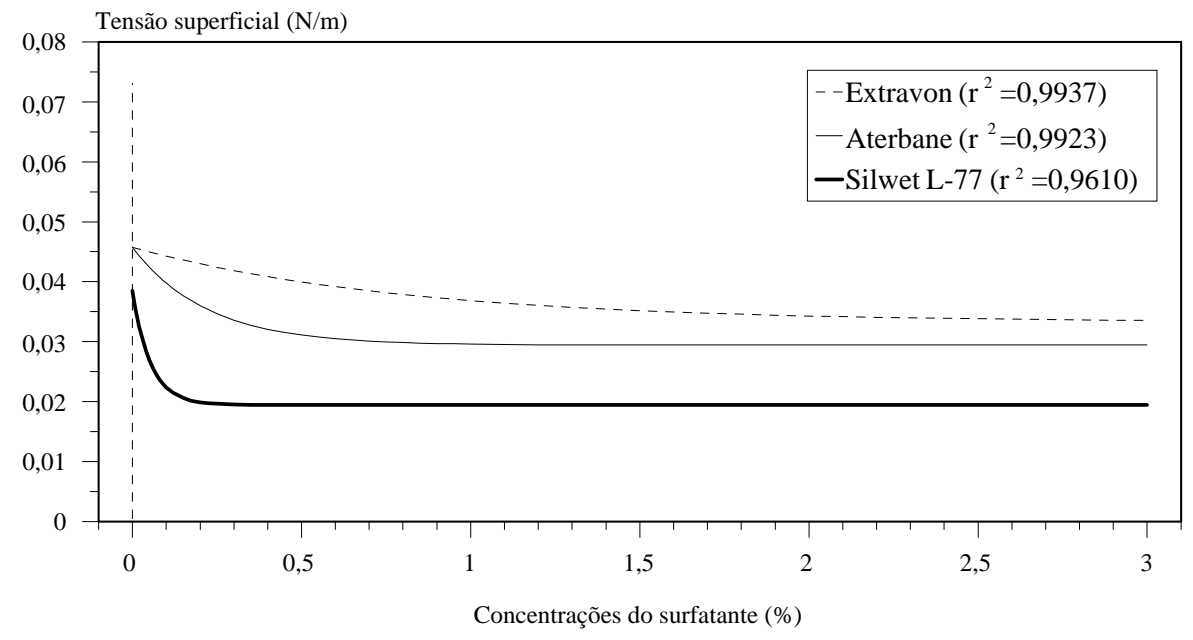

FIGURA 5. Tensões superficiais em função da concentração dos surfatantes Extravon, Aterbane e Silwet L-77. Concentração de glyphosate: $3,5 \%$ do produto comercial Roundup. Estimativas segundo o modelo de Mitscherlich. 


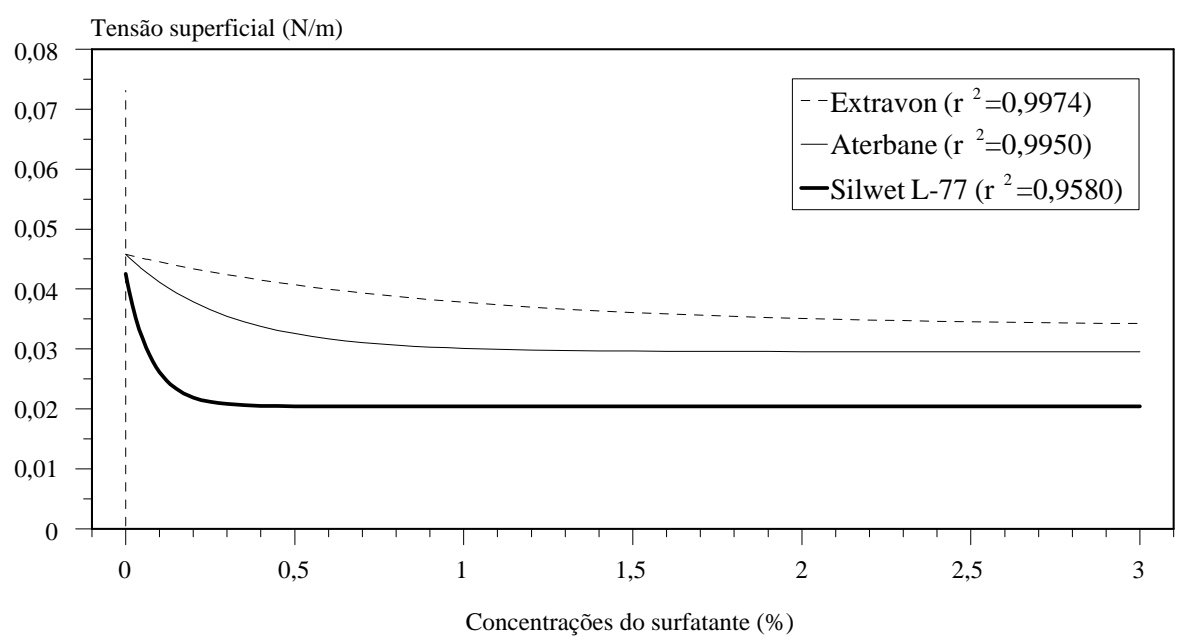

FIGURA 6. Tensões superficiais em função da concentração dos surfatantes Extravon, Aterbane e Silwet L-77. Concentração de glyphosate: $5 \%$ do produto comercial Roundup. Estimativas segundo o modelo de Mitscherlich.

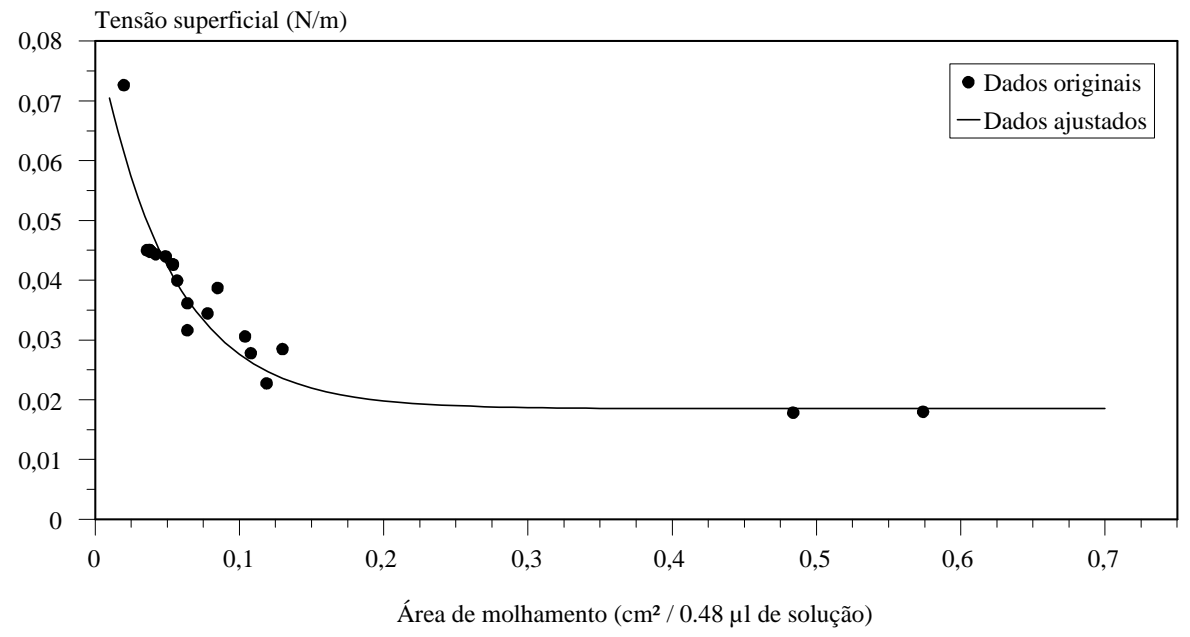

FIGURA 7. Área de folha molhada por $0,48 \mu$ de soluções de glyphosate e dos surfatantes Extravon, Aterbane e Silwet L-77, em função da tensão superficial. Estimativas segundo o modelo de Mitscherlich. 
TABELA 3. Valores médios da tensão superficial $(\mathrm{mN} / \mathrm{m})$ e os valores correspondentes de área de molhamento $\left(\mathrm{cm}^{2} / 0,48 \mu \mathrm{l}\right)$.

\begin{tabular}{|c|c|c|c|c|c|c|c|}
\hline \multirow{2}{*}{$\begin{array}{l}\text { Concen- } \\
\text { tração de } \\
\text { Roundup }\end{array}$} & \multirow{2}{*}{$\begin{array}{l}\text { Concen- } \\
\text { tração do } \\
\text { surfatante }\end{array}$} & \multicolumn{2}{|c|}{ Extravon } & \multicolumn{2}{|c|}{ Aterbane } & \multicolumn{2}{|c|}{ Silwet L-77 } \\
\hline & & $\begin{array}{c}\text { Tensão } \\
\text { superficial } \\
(\mathrm{mN} / \mathrm{m}) \\
\end{array}$ & $\begin{array}{c}\text { Área de } \\
\text { molhamento } \\
\left(\mathrm{cm}^{2} / 0,48 \mu \mathrm{l}\right)\end{array}$ & $\begin{array}{c}\text { Tensão } \\
\text { superficial } \\
(\mathrm{mN} / \mathrm{m}) \\
\end{array}$ & $\begin{array}{c}\text { Área de } \\
\text { molhamento } \\
\left(\mathrm{cm}^{2} / 0,48 \mu \mathrm{l}\right)\end{array}$ & $\begin{array}{c}\text { Tensão } \\
\text { superficial } \\
(\mathrm{mN} / \mathrm{m}) \\
\end{array}$ & $\begin{array}{c}\text { Área de } \\
\text { molhamento } \\
\left(\mathrm{cm}^{2} / 0,48 \mu \mathrm{l}\right)\end{array}$ \\
\hline 0 & 0 & 72,6 & 0,02 & 72,6 & 0,02 & 72,6 & 0,02 \\
\hline 1 & 0 & 45,0 & 0,036 & 45,0 & 0,036 & 45,0 & 0,036 \\
\hline 2 & 0 & 44,7 & 0,038 & 44,7 & 0,038 & 44,7 & 0,038 \\
\hline 3,5 & 0 & 44,3 & 0,042 & 44,3 & 0,042 & 44,3 & 0,042 \\
\hline 5 & 0 & 44,0 & 0,049 & 44,0 & 0,049 & 44,0 & 0,049 \\
\hline 2 & 0,005 & - & - & - & - & 38,7 & 0,085 \\
\hline 2 & 0,02 & - & - & 42,5 & 0,054 & 30,5 & 0,104 \\
\hline 2 & 0,05 & 42,6 & 0,054 & - & - & - & - \\
\hline 2 & 0,1 & - & - & 36,1 & 0,064 & 22,7 & 0,119 \\
\hline 2 & 0,2 & 39,9 & 0,057 & - & - & - & - \\
\hline 2 & 1 & 34,5 & 0,078 & 28,4 & 0,130 & 17,9 & 0,574 \\
\hline 2 & 3 & 31,6 & 0,064 & 27,8 & 0,108 & 17,8 & 0,484 \\
\hline
\end{tabular}

- não foi estudado.

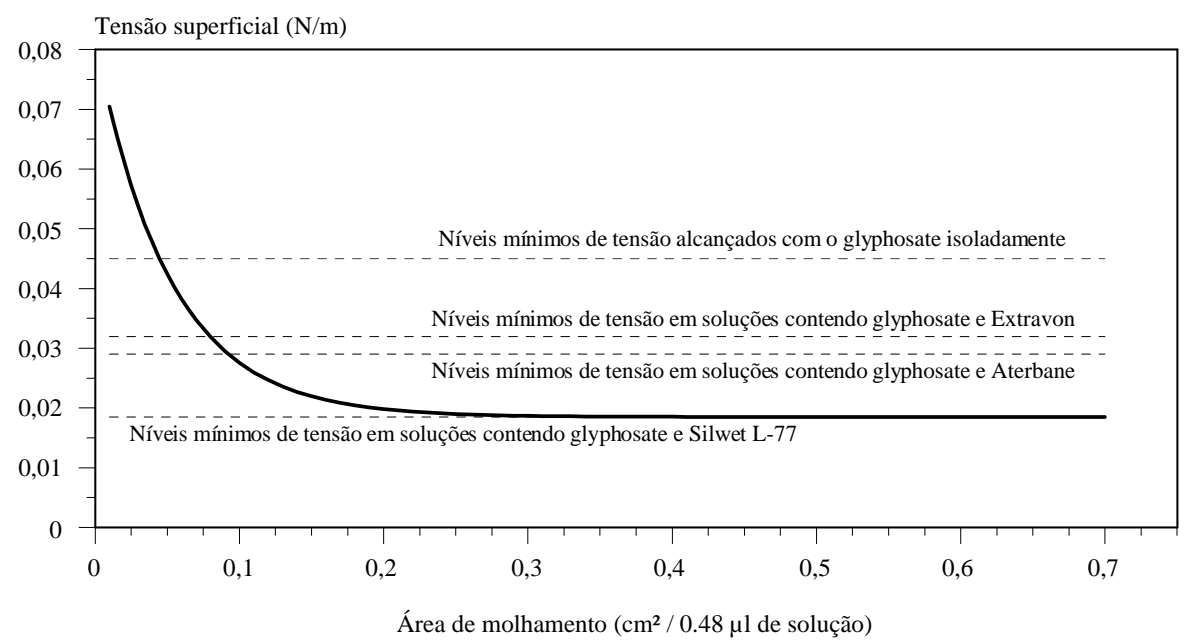

FIGURA 8. Área de folha molhada por $0,48 \mu$ de soluções de glyphosate e dos surfatantes Extravon, Aterbane e Silwet L-77, em função da tensão superficial. Estimativas segundo o modelo de Mitscherlich. 


\section{AGRADECIMENTOS}

Agradecimento ao $\mathrm{CNPq} / \mathrm{PIBIC}$ pela concessão de bolsa de Iniciação Científica ao primeiro autor para execução deste trabalho.

\section{LITERATURA CITADA}

BAYLIS, A. D., HART C. A. Varying responses among weed species to glyphosatetrimesium in the presence of an organosilicone surfactant. Bringhton Crop Protection Conference - Weeds, p.1331-6, 1993.

BUICK, R. D., BUCHAN, G. D., FIELD, R. J. The role of surface tennsion of spreading droplets in absorption of a herbicide formulation via leaf stomata. Pestic. Sci., v.38, n.2\&3, p.227-35, 1993.

CHUNG, B. J., KWON, Y. W. Relationship between surfactant properties and wettability of rice leaf surfaces for several nonionic surfactants. In: FOY, C. L., Adjuvants for agrochemicals. Florida: CRC Press, 1992. p.37-58.

GREENE, D. W., BUKOVAC, M. J. Stomatal penetration: effect of surfactants and role in foliar absorption. Amer. J. Bot., v.61, n.1, p.101-6, 1974.

GODDARD, E. D., PADMANABHAN, K. P. A. A mechanistic study of the wetting, spreading, and solution properties of organosilicone surfactants. In: FOY, C. L., Adjuvants for agrochemicals. Florida: CRC Press, 1992. p.373-83.
KADOTA, G., MATSUNAKA, S. Effects of surfactants on foliar wettability in rice plants. Journ. of Pest. Sci, v.11, p.597603, 1986.

REDDY, K. N., SINGH, M. Organosilicone adjuvants effects on glyphosate efficacy and rainfastness. Weed Technol., v.6, n.2, p.361-5, 1992.

ROGGENBUCK, F. C., ROWE, L., PENNER, D., PETROF, L., BUROW, R. Increasing postemergence herbicide efficacy and rainfastness with silicone adjuvants. Weed Technol., v.4, n.3, p.576-80, 1990.

SINGH, M., MACK, R. E. Effect of organosilicone-based adjuvants on herbicide efficacy. Pestic. Sci., v.38, n.2\&3, p.219-25, 1993.

STEVENS, P. J. G., KIMBERLEY, M. O., MURPHY, D. S., POLICELLO, G. A. Adhesion of spray droplets to foliage: the role of dynamic surface tension and advantages of organosilicone surfactants. Pestic. Sci., v.38, n.2\&3, p. 237-45, 1993.

STOCK, D., HOLlOWAY, P. J. Possible mechanisms for surfactant-induced foliar uptake of agrochemicals. Pestic. Sci, v.38, n.2\&3, p.165-77, 1993.

SUN, J., FOY, C. L., WITT, H. L. Effect of organosilicone surfactants on the rainfastness of primisulffuron in velvetleaf (Abutilon theophrasti). Weed Technol., v.10, n.2, p.263-7, 1996. 